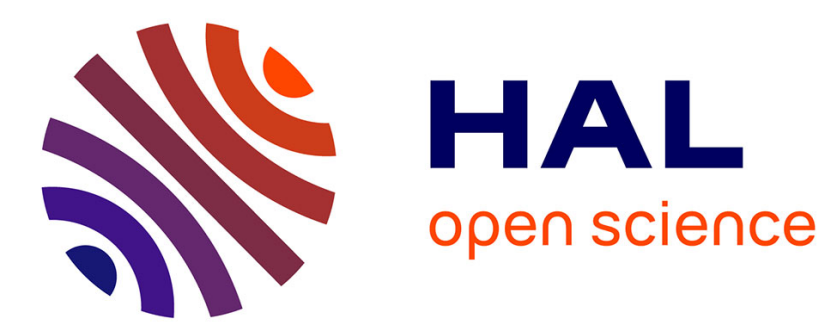

\title{
Quinze Ans Après...
}

Christian Licht, Thibaut Weller

\section{To cite this version:}

Christian Licht, Thibaut Weller. Quinze Ans Après.... Frémond Michel; Maceri Franco; Vairo Giuseppe. Models, Simulation, and Experimental Issues in Structural Mechanics, Springer International Publishing, pp.41-60, 2017, 978-3-319-48884-4. 10.1007/978-3-319-48884-4_2 . hal-01493410

\section{HAL Id: hal-01493410 https://hal.science/hal-01493410}

Submitted on 21 Mar 2017

HAL is a multi-disciplinary open access archive for the deposit and dissemination of scientific research documents, whether they are published or not. The documents may come from teaching and research institutions in France or abroad, or from public or private research centers.
L'archive ouverte pluridisciplinaire HAL, est destinée au dépôt et à la diffusion de documents scientifiques de niveau recherche, publiés ou non, émanant des établissements d'enseignement et de recherche français ou étrangers, des laboratoires publics ou privés. 


\title{
Quinze ans après...
}

\author{
Christian Licht $\dagger \ddagger$, Thibaut Weller $\dagger$ \\ $\dagger$ Laboratoire de Mécanique et Génie Civil, UMR 5508 CNRS-UM 2, Université \\ Montpellier II, c.c. 48, Place Eugène Bataillon, 34095 Montpellier cedex 05, France \\ (e-mail: firstname.lastname@umontpellier.fr) \\ $\ddagger$ Department of Mathematics, Faculty of Science, Mahidol University, Bangkok 10400, \\ Thailand
}

\begin{abstract}
We aim to present mathematical models of smart devices and smart structures. Smart devices are made of materials which present significant multiphysical couplings. They are integrated in smart structures which take technological advantages of some multiphysical effects. We first propose simplified but accurate models of thin plates or slender rods made of piezoelectric or electromagneto-elastic materials in both static and dynamic cases. Then we focus on smart structures such as piezoelectric patches bonded on a linearly elastic body and piezoelectric junctions between two linearly piezoelectric or elastic bodies.
\end{abstract}

Keywords: Asymptotic analysis, plates and rods models, piezoelectricity, patches and junctions.

\section{Introduction}

On November 2000, Franco Maceri came to the LMGC to present the next Colloquium Lagrangianum in Taormina and a recent study [2] on piezoelectric plates. Here we present all the studies about mathematical modeling in piezoelectricity (a topic totally new for us) we did after this stimulating talk.

In the first part we intend to propose simplified but accurate models of devices made of piezoelectric or elecromagneto-elastic materials, these devices (thin plates, slender rods) presenting one or two small dimensions. We also studied the not so well-known case of piezoelectricity with electric field gradient. The models are obtained by a rigorous study of the asymptotic behavior of a three dimensional body when some of its dimensions, considered as parameters, tend to zero. We used various tools of variational and functional analysis, the point being to consider boundary value problems depending on small parameters. This study has been carried out in the steady-state and transient cases. We outline that different kinds of models appear at the limit, depending on the electrical loading. These models correspond to the physical situation when the device behaves as a sensor or as an actuator. Moreover, we are able to show that depending on the crystalline symmetry class of the material, a striking structural switch-off may appear at the limit, the device being no more piezoelectric.

The essential technological interest of piezoelectric devices being the monitoring of a deformable body they are bonded to or integrated in, the second part is devoted to smart structures. The obtained results are discussed in detail in Section 3. 
Of course, this field of research has led in the past twenty years to a considerable amount of literature. In this paper, we limit ourselves to our own work. The reader will find in the references of our studies a great number of articles published in this area.

\section{Mathematical modelings of smart devices}

As usual we make no difference between the physical space and $\mathbb{R}^{3}$ whose basis is denoted $\left(e_{1}, e_{2}, e_{3}\right)$. For all $\xi=\left(\xi_{1}, \xi_{2}, \xi_{3}\right)$ in $\mathbb{R}^{3}$, we denote $\left(\xi_{1}, \xi_{2}\right)$ by $\widehat{\xi}$. Greek indices for coordinates take their values in $\{1,2\}$ whereas latin indices run from 1 to 3 .

Let $\mathbb{H}=\mathbb{S}^{3} \times \mathbb{R}^{3}$, where $\mathbb{S}^{3}$ denotes the set of all $3 \times 3$ real and symmetric matrices. The set of all linear mappings from a space $V$ into a space $W$ is denoted $\mathcal{L}(V, W)$ and by $\mathcal{L}(V)$ if $V=W$.

In the sequel, for every domain $G$ in $\mathbb{R}^{N}$, the subspace of the Sobolev space $H^{1}(G)$ whose elements vanish on $\Gamma$, included in the boundary $\partial G$ of $G$, will be denoted by $H_{\Gamma}^{1}(G)$.

\subsection{Piezoelectric thin plates}

Finding the equilibrium of a thin linearly piezoelectric plate can be formulated as follows. The reference configuration of a linearly piezoelectric thin plate is the closure in $\mathbb{R}^{3}$ of the set $\Omega^{\varepsilon}:=\omega \times(-\varepsilon, \varepsilon)$, where $\omega$ is a bounded domain of $\mathbb{R}^{2}$ with Lipschitz boundary $\partial \omega$ and $\varepsilon$ a small positive number. Let $\Gamma_{\text {lat }}^{\varepsilon}:=\partial \omega \times(-\varepsilon, \varepsilon), \Gamma_{ \pm}^{\varepsilon}:=\omega \times\{ \pm \varepsilon\}$ and two suitable partitions of $\partial \Omega^{\varepsilon}:\left(\Gamma_{m D}^{\varepsilon}, \Gamma_{m N}^{\varepsilon}\right)$ and $\left(\Gamma_{e D}^{\varepsilon}, \Gamma_{e N}^{\varepsilon}\right)$ with $\Gamma_{m D}^{\varepsilon}$ and $\Gamma_{e D}^{\varepsilon}$ of strictly positive surface measures. The plate is clamped along $\Gamma_{m D}^{\varepsilon}$ and at an electrical potential $\varphi_{0}^{\varepsilon}$ on $\Gamma_{e D}^{\varepsilon}$. It is subjected to body forces $f^{\varepsilon}$ in $\Omega^{\varepsilon}$ and to surface forces $g^{\varepsilon}$ in $\Gamma_{m N}^{\varepsilon}$. Furthermore, we will consider an electrical loading $d^{\varepsilon}$ on $\Gamma_{e N}^{\varepsilon}$. We note $n^{\varepsilon}$ the outward unit normal to $\partial \Omega^{\varepsilon}$ and assume that $\Gamma_{m D}^{\varepsilon}=\gamma_{0} \times(-\varepsilon, \varepsilon)$, with $\gamma_{0} \subset \partial \omega$. The equations determining the piezoelectric state $s^{\varepsilon}:=\left(u^{\varepsilon}, \varphi^{\varepsilon}\right)$ at equilibrium are:

$$
\mathcal{P}\left(\Omega^{\varepsilon}\right)\left\{\begin{array}{l}
\operatorname{div} \sigma^{\varepsilon}+f^{\varepsilon}=0 \text { in } \Omega^{\varepsilon}, \sigma^{\varepsilon} n^{\varepsilon}=g^{\varepsilon} \text { on } \Gamma_{m N}^{\varepsilon}, u^{\varepsilon}=0 \text { on } \Gamma_{m D}^{\varepsilon}, \\
\operatorname{div} D^{\varepsilon}=0 \text { in } \Omega^{\varepsilon}, D^{\varepsilon} \cdot n^{\varepsilon}=d^{\varepsilon} \text { on } \Gamma_{e N}^{\varepsilon}, \varphi^{\varepsilon}=\varphi_{0}^{\varepsilon} \text { on } \Gamma_{e D}^{\varepsilon}, \\
\left(\sigma^{\varepsilon}, D^{\varepsilon}\right)=M^{\varepsilon}(x)\left(e\left(u^{\varepsilon}\right), \nabla \varphi^{\varepsilon}\right) \text { in } \Omega^{\varepsilon}
\end{array}\right.
$$

where $u^{\varepsilon}, \varphi^{\varepsilon}, \sigma^{\varepsilon}, e\left(u^{\varepsilon}\right)$ and $D^{\varepsilon}$ respectively stand for the displacement, the electrical potential, the stress tensor, the tensor of small strains (i.e. the symmetrized gradient) and the electric induction. The operator $M^{\varepsilon}$ is an element of $\mathcal{L}(\mathcal{H})$ such that:

$$
\begin{aligned}
& \sigma^{\varepsilon}=a^{\varepsilon} e\left(u^{\varepsilon}\right)-b^{\varepsilon} \nabla \varphi^{\varepsilon} \\
& D^{\varepsilon}=b^{\varepsilon^{T}} e\left(u^{\varepsilon}\right)+c^{\varepsilon} \nabla \varphi^{\varepsilon}
\end{aligned}
$$

with $b^{\varepsilon^{T}}$ the transpose of the piezoelectric tensor $b^{\varepsilon}$, the elastic tensor $a^{\varepsilon}$ and the dielectric one $c^{\varepsilon}$ being symmetric and positive. Note that because of the piezoelectric coupling, $M^{\varepsilon}$ is not symmetric.

It is easy to give a weak (or variational) formulation of the previous linear boundary problem and to conclude to the existence and the uniqueness of a solution in suitable Sobolev spaces through the Stampacchia theorem.

Nevertheless, due to the very low thickness of the plate, this classical model may be difficult to tackle numerically. The essence of our proposal of simplified but accurate modeling is to consider $\varepsilon$ as a small parameter and to study the asymptotic behavior of $s^{\varepsilon}$ when $\varepsilon$ goes to 0 . In fact, two different limit behaviors indexed by $p \in\{1,2\}$ will occur, according to the type of boundary condition in $\mathcal{P}\left(\Omega^{\varepsilon}\right)$. 
From the mathematical point of view it is convenient to proceed to a change of coordinates $\Pi^{\varepsilon}$ and of unknows $s_{p}(\varepsilon)=S_{p}(\varepsilon) s^{\varepsilon}$ in order to consider functional spaces defined on a fixed domain $\Omega=\omega \times(-1,1)$.

$$
\begin{aligned}
x=\left(x_{1}, x_{2}, x_{3}\right) \in \bar{\Omega} & \mapsto \Pi^{\varepsilon} x=\left(x_{1}, x_{2}, \varepsilon x_{3}\right) \in \bar{\Omega}^{\varepsilon} \\
s_{p}(\varepsilon):=\left(u(\varepsilon)(x), \varphi_{p}(\varepsilon)(x)\right) & =\left(\left(\varepsilon^{-1} \hat{u}^{\varepsilon}\left(\Pi^{\varepsilon} x\right), u_{3}^{\varepsilon}\left(\Pi^{\varepsilon} x\right)\right), \varepsilon^{-p} \varphi^{\varepsilon}\left(\Pi^{\varepsilon} x\right)\right) .
\end{aligned}
$$

The formulae defining $S_{p}(\varepsilon)$ stem from the assumptions on the magnitude of the electromechanical loading and are justified by the convergence results they lead to. If we consider forces and displacements, these hypotheses are the ones of [3] and supply a mathematical justification of the Kirchhoff-Love theory of thin linearly elastic plates. In addition, we assume that $\varphi_{0}^{\varepsilon}$ has an extension into $\Omega^{\varepsilon}$ still denoted by $\varphi_{0}^{\varepsilon}$ and that $\varphi_{0} \in H^{1}(\Omega)$ is such that $\varphi_{0}^{\varepsilon}\left(\Pi^{\varepsilon} x\right)=\varepsilon^{p} \varphi_{0}(x)$ with:

$$
\left\{\begin{aligned}
\text { if } p=1: & \varphi_{0} \text { does not depend on } x_{3} . \\
\text { if } p=2: & \text { the closure of the projection of } \Gamma_{e D}^{\varepsilon} \text { on } \omega \text { coincides with } \bar{\omega}, \\
& \text { moreover, either } d^{\varepsilon}=0 \text { on } \Gamma_{e N}^{\varepsilon} \cap \Gamma_{\text {lat }}^{\varepsilon} \text { or } \Gamma_{e N}^{\varepsilon} \cap \Gamma_{\text {lat }}^{\varepsilon}=\emptyset .
\end{aligned}\right.
$$

Thus $s(\varepsilon)$ is the solution of the variational problem:

$$
\left\{\begin{array}{l}
\text { Find } s_{p}(\varepsilon) \in\left(0, \varphi_{0}\right)+V=\left\{r=(v, \psi) \in H_{\Gamma_{m D}}^{1}(\Omega)^{3} \times H_{\Gamma_{e D}}^{1}(\Omega)\right\} \text { such that } \\
\int_{\Omega} M(x) k_{p}(\varepsilon, s) \cdot k_{p}(\varepsilon, r) d x=L(r), \forall r \in V
\end{array}\right.
$$

where the linear form $L$ does not depend on $\varepsilon$ and

$$
\left\{\begin{array}{l}
k_{p}(\varepsilon, r)=k_{p}(\varepsilon,(v, \psi))=\left(e(\varepsilon, v), \nabla_{p}(\varepsilon, \psi)\right) \\
e(\varepsilon, v)_{\alpha \beta}=e(v)_{\alpha \beta}, e(\varepsilon, v)_{\alpha 3}=\varepsilon^{-1} e(v)_{\alpha 3}, e(\varepsilon, v)_{33}=\varepsilon^{-2} e(v)_{33}, \\
\widehat{\nabla}_{p}(\varepsilon, \psi)=\varepsilon^{p-1} \widehat{\nabla} \psi, \nabla_{p}(\varepsilon, \psi)_{3}=\varepsilon^{p-2} \partial_{3} \psi
\end{array}\right.
$$

The signs of the various powers of $\varepsilon$ in the components of $k_{p}(\varepsilon, r)$ induce an orthogonal decomposition of $\mathbb{H}$ in subspaces $\mathbb{H}_{p}^{\star}$, with $\star \in\{-, 0,+\}$, which is crucial to fully describe plates models in all admissible crystal classes. We denote by $h_{p}^{\star}$ the projection on $\mathbb{H}_{p}^{\star}$ of any element $h$ of $\mathbb{H}$ so that $M$ can then be decomposed in nine elements $M_{p}^{\star \diamond} \in \mathcal{L}\left(\mathbb{H}_{p}^{\diamond}, \mathbb{H}_{p}^{\star}\right)$, with $\star, \diamond \in\{-, 0,+\}$. Because $M_{p}^{00}$ and $M_{p}^{--}$are positive operators on $\mathbb{H}_{p}^{0}$ and $\mathbb{H}_{p}^{-}$, the Schur complement

$$
\widetilde{M}_{p}:=M_{p}^{00}-M_{p}^{0-}\left(M_{p}^{--}\right)^{-1} M_{p}^{-0}
$$

is an element of $\mathcal{L}\left(\mathbb{H}_{p}^{0}\right)$. The key point of the asymptotic study is to show that if $\bar{k}_{p}$ is the limit (in a suitable topology) of $k_{p}\left(\varepsilon, s_{p}(\varepsilon)\right)$, then $\left(M \bar{k}_{p}\right)_{p}^{-}=\left(\bar{k}_{p}\right)_{p}^{+}=0$. This will enable us to exhibit $\widetilde{M}_{p}$ as the operator governing the limit constitutive equations due to the fundamental relation:

$$
(M h)_{p}^{-}=h_{p}^{+}=0 \Rightarrow \widetilde{M}_{p} h_{p}^{0}=(M h)_{p}^{0} \text { and } \widetilde{M}_{p} h_{p}^{0} \cdot h_{p}^{0}=M h \cdot h .
$$

The limit space of displacements will be the space of Kirchhoff-Love displacements defined by $\mathbf{V}_{K L}:=\left\{v \in H_{\Gamma_{m D}}^{1}(\Omega)^{3} ; e_{i 3}(v)=0\right\}$ while the limit electrical spaces will be $\Phi_{e, 1}:=\left\{\psi \in H_{\Gamma_{e D}}^{1}(\Omega) ; \partial_{3} \psi=0\right\}$ and $\Phi_{e, 2}:=\left\{\psi \in H_{\partial_{3}}^{1}(\Omega) ; \psi_{\mid \Gamma_{e D} \cap \Gamma^{ \pm}}=0\right\}$, where 
$H_{\partial_{3}}^{1}(\Omega):=\left\{\psi \in L^{2}(\Omega) ; \partial_{3} \psi \in L^{2}(\Omega)\right\}$ and where $\Gamma_{e D}$ stands for the image of $\Gamma_{e D}^{\varepsilon}$ by $\Pi^{\varepsilon^{-1}}$. Finally, we have the following convergence result:

Let $\boldsymbol{K}_{1}:=H^{1}(\Omega)$ and $\boldsymbol{K}_{2}:=H_{\partial_{3}}^{1}(\Omega)$. When $\varepsilon \rightarrow 0$, the familly $\left(s_{p}(\varepsilon)\right)_{\varepsilon>0}$ of the unique solutions of $\mathcal{P}(\varepsilon, \Omega)_{p}$ strongly converges in $\boldsymbol{X}_{p}:=H_{\Gamma_{m D}}^{1}(\Omega)^{3} \times \boldsymbol{K}_{p}$ to the unique solution $\bar{s}_{p}$ of

$$
\overline{\mathcal{P}}(\Omega)_{p}\left\{\begin{array}{l}
\text { Find } s \in\left(0, \varphi_{0}\right)+\mathbf{S}_{p} \text { such that } \\
\int_{\Omega} \widetilde{M}_{p} k(s)_{p}^{0} \cdot k(r)_{p}^{0} d x=L(r), \forall r \in \mathbf{S}_{p}:=\mathbf{V}_{K L} \times \Phi_{e, p} .
\end{array}\right.
$$

To get physically meaningful results, we define an electromechanical state $\bar{s}_{p}^{\varepsilon}$ over the real plate $\Omega^{\varepsilon}$ by the descaling $\bar{s}_{p}^{\varepsilon}=S_{p}(\varepsilon)^{-1} \bar{s}_{p}$ : it is the unique solution of a problem $\overline{\mathcal{P}}\left(\Omega^{\varepsilon}\right)_{p}$ posed over $\Omega^{\varepsilon}$ which is the transportation by $\Pi^{\varepsilon}$ of the (limit scaled) problem $\overline{\mathcal{P}}(\Omega)_{p}$. This transported problem is our proposal to model the thin linearly piezoelectric plate of thickness $2 \varepsilon$. Our model in fact involves two dimensional problems set on $\omega$, which is very attractive and favourable from the numerical point of view. It is also accurate in the sense that the convergence result on the scaled states implies that $s^{\varepsilon}$ is asymptotically equivalent to $\bar{s}_{p}^{\varepsilon}$.

The first model $(p=1)$ with $\varphi_{0}=0$ deals with the physical situation when the plate is used as a sensor, the second model corresponds to an actuator. The model involves "reduced" state variables, the sole component $k_{p}^{0}$ of the couple strain/gradient of the electrical potential, and the constitutive equation are supplied by the Schur complement (or the "condensation" of the initial operator $M^{\varepsilon}$ ) with respect to the maintained components. This identification is the keypoint for obtaining some decoupling and symmetry properties very important in practice (see [20], [19] and [4]) by due account of the influence of the crystalline symmetries on the coefficients of $\widetilde{M}_{p}$. More precisely, it is possible to list some properties of the operator $\widetilde{M}_{p}(p=1,2)$, which supplies the constitutive equations of the piezoelectric plate.

The fundamental coupling property of $M$ remains true for $\widetilde{M}_{p}$ :

$$
\widetilde{M}_{p_{m e}}=-\left(\widetilde{M}_{p_{e m}}\right)^{T},
$$

where $m$ and $e$ respectively denote the mechanical and electrical components of the generalized kinematics and stresses.

Considering the influence of crystalline symmetries on the three-dimensional constitutive law (see [13] for example), we can deduce, in the case of a polarization normal to the plate, that:

- $\widetilde{M}_{2_{m m}}$ involves mechanical terms only,

- $\widetilde{M}_{1_{m m}}=\widetilde{M}_{2_{m m}}$ for the crystalline classes $m, 32,422, \overline{6}, 622$ and $\overline{6} m 2$,

- $\widetilde{M}_{1_{m m}}$ involves electrical terms except for these previous classes,

- when $p=1$, there is an electromechanical decoupling $\left(\widetilde{M}_{p_{m e}}=0\right)$ for the classes 2 , $222,2 \mathrm{~mm}, 4, \overline{4}, 422,4 \mathrm{~mm}, \overline{4} 2 \mathrm{~m}, 6,622,6 \mathrm{~mm}$ and 23 , when $p=2$, this decoupling occurs with the classes $m, 32,422, \overline{6}, 622$ and $\overline{6} m 2$, nevertheless the operators $\widetilde{M}_{p_{m m}}$ and $\widetilde{M}_{p_{e e}}$ involve a mixture of elastic, piezoelectric and dielectric coefficients. In these cases, the plate can be considered as no more piezoelectric. We are then in a situation of a structural switch-off of the piezoelectric effect. 
Let us consider for example a thin piezoelectric plate constituted by a material whose crystalline symmetry class is 222 . Then (1) takes the following form:

$$
\left(\begin{array}{c}
\sigma_{11} \\
\sigma_{22} \\
\sigma_{33} \\
\sqrt{2} \sigma_{23} \\
\sqrt{2} \sigma_{31} \\
\sqrt{2} \sigma_{12} \\
D_{1} \\
D_{2} \\
D_{3}
\end{array}\right)=\left(\begin{array}{cccccc|ccc}
a_{11} & a_{12} & a_{13} & 0 & 0 & 0 & 0 & 0 & 0 \\
a_{12} & a_{22} & a_{23} & 0 & 0 & 0 & 0 & 0 & 0 \\
a_{13} & a_{23} & a_{33} & 0 & 0 & 0 & 0 & 0 & 0 \\
0 & 0 & 0 & a_{44} & 0 & 0 & -b_{41} & 0 & 0 \\
0 & 0 & 0 & 0 & a_{55} & 0 & 0 & -b_{52} & 0 \\
0 & 0 & 0 & 0 & 0 & a_{66} & 0 & 0 & -b_{63} \\
\hline 0 & 0 & 0 & b_{41} & 0 & 0 & c_{11} & 0 & 0 \\
0 & 0 & 0 & 0 & b_{52} & 0 & 0 & c_{22} & 0 \\
0 & 0 & 0 & 0 & 0 & b_{63} & 0 & 0 & c_{33}
\end{array}\right) \cdot\left(\begin{array}{c}
e_{11}(u) \\
e_{22}(u) \\
e_{33}(u) \\
\sqrt{2} e_{23}(u) \\
\sqrt{2} e_{31}(u) \\
\sqrt{2} e_{12}(u) \\
\varphi, 1 \\
\varphi_{, 2} \\
\varphi_{, 3}
\end{array}\right) .
$$

Therefore, (5) leads to

$$
\widetilde{M}_{1}=\left(\begin{array}{ccc|cc}
\frac{a_{11} a_{33}-a_{13}^{2}}{a_{33}} & \frac{a_{12} a_{33}-a_{13} a_{23}}{a_{33}} & 0 & 0 & 0 \\
\frac{a_{12} a_{33}-a_{13} a_{23}}{a_{33}} & \frac{a_{22} a_{33}-a_{23}^{2}}{a_{33}} & 0 & 0 & 0 \\
0 & 0 & \frac{a_{66} c_{33}+b_{63}^{2}}{c_{33}} & 0 & 0 \\
\hline 0 & 0 & 0 & \frac{c_{11} a_{44}+b_{41}^{2}}{a_{44}} & 0 \\
0 & 0 & 0 & 0 & \frac{c_{22} a_{55}+b_{52}^{2}}{a_{5}}
\end{array}\right)
$$

in the sensor case and to

$$
\widetilde{M}_{2}=\left(\begin{array}{ccc|c}
\frac{a_{11} a_{33}-a_{13}^{2}}{a_{33}} & \frac{a_{12} a_{33}-a_{13} a_{23}}{a_{33}} & 0 & 0 \\
\frac{a_{12} a_{33}-a_{13} a_{23}}{a_{33}} & \frac{a_{22} a_{33}-a_{23}^{2}}{a_{33}} & 0 & 0 \\
0 & 0 & a_{66} & -b_{63} \\
\hline 0 & 0 & b_{63} & c_{33}
\end{array}\right)
$$

in the actuator case.

As outlined previsouly, the relation (9) shows that $\sigma$ and $D$ respectively depend only on $e(u)$ and $\nabla \varphi$ when the plate acts as a sensor, so that it can be considered as no more piezoelectric. But, when the same plate acts as an actuator, the piezoelectric coupling does not vanishes as it can be seen in (10).

\section{$2.2 \quad$ Electromagneto-elastic thin plates}

Besides the piezoelectric coupling, some materials are sensitive to magnetic effects, thus in [22] we extended the previous modeling to linearly electromagneto-elastic thin plates. Now the state is described by $s^{\varepsilon}=\left(u^{\varepsilon}, \varphi^{\varepsilon}, \phi^{\varepsilon}\right)$ where the additional variable $\phi^{\varepsilon}$ denotes the magnetic potential and the constitutive equations read as:

$$
\begin{aligned}
& \sigma^{\varepsilon}=a^{\varepsilon} e\left(u^{\varepsilon}\right)-b^{\varepsilon} \nabla \varphi^{\varepsilon}-d^{\varepsilon} \nabla \phi^{\varepsilon} \\
& D^{\varepsilon}=b^{\varepsilon^{T}} e\left(u^{\varepsilon}\right)+c^{\varepsilon} \nabla \varphi^{\varepsilon}+e^{\varepsilon} \nabla \phi^{\varepsilon}, \\
& B^{\varepsilon}=d^{\varepsilon^{T}} e\left(u^{\varepsilon}\right)+e^{\varepsilon^{T}} \nabla \varphi^{\varepsilon}+f^{\varepsilon} \nabla \phi^{\varepsilon} .
\end{aligned}
$$

In these constitutive equations, $d^{\varepsilon}, e^{\varepsilon}$ and $f^{\varepsilon}$ respectively stand for the piezomagnetic, electromagnetic coupling and magnetic permeability tensors, while $B^{\varepsilon}$ denotes the magnetic induction. 
A similar mathematical analysis of the asymptotic behavior of $s^{\varepsilon}$ can be done to derive a simplified but accurate model of thin electromagneto-elastic plate. It involves reduced state variable and constitutive equations supplied by the condensation $\widetilde{M^{\varepsilon}}$ of $M^{\varepsilon}$ with respect to the maintained components of $\left(e\left(u^{\varepsilon}\right), \nabla \varphi^{\varepsilon}, \nabla \phi^{\varepsilon}\right)$.

But the novelty here is that four limit behaviors may appear according to the type of boundary conditions and the magnitude of the data on the electric and magnetic fields. These cases can be described as previously but by a couple of indices $(p, q) \in\{1,2\}^{2}$ in place of the sole indice $p$. The physical situation when the thin plate is used as an electrical (resp. magnetic) sensor corresponds to $p=1$ (resp. $q=1$ ) while the actuator case corresponds to $p, q=2$. It therefore appears two original mixed behaviors when $p \neq q$. In these situations, the plate is at the same time a sensor and an actuator excepted for the classes for which the plate is no more electromagneto-elastic (i.e. the electromechanical and magnetomechanical coefficients in $\widetilde{M}^{\varepsilon}$ vanishes). The two cases $p \neq q$ allow the modeling of electrically commanded magnetic devices and of magnetically commanded electric ones, which is of considerable interest in the development of non-volatile magnetic random access memories. We emphasize on the point that this behavior is here fully described for any admissible crystal class.

\subsection{Piezoelectric plates with electric field gradient}

In the 1960's the study of unexplained aspects of piezoelectricity led Mindlin [10] to extend the classical Voigt theory [17] in Toupin's formulation [15] by assuming that the stored energy function not only depends on the strain tensor and polarization vector but also on the polarization gradient tensor. What motivated Mindlin to study the effects of the polarization gradient was the capacitance of a very thin dielectric film. Experiments showed that the capacitance of a very thin film is systematically smaller than the classical prediction. Moreover, performing experimental tests, Mead [9] showed that piezoelectric effects can also appear in centrosymmetric crystals, which is in contradiction with classical Voigt theory. And, indeed, the Mindlin's theory of elastic dielectrics with polarization gradient accomodates the observed and experimentally measured phenomena, such as electromechanical interactions in centrosymmetric materials, capacitance of thin dielectric films, surface energy of polarization, deformation and optical activity in quartz.

As in the classical piezoelectric case the physical state is described by $s^{\varepsilon}=\left(u^{\varepsilon}, \varphi^{\varepsilon}\right)$. However, the constitutive equations read as:

$$
\begin{aligned}
& \sigma^{\varepsilon}=a^{\varepsilon} e\left(u^{\varepsilon}\right)-b^{\varepsilon} \nabla \varphi^{\varepsilon}-\alpha^{\varepsilon} \nabla^{2} \phi^{\varepsilon}, \\
& D^{1, \varepsilon}=b^{\varepsilon^{T}} e\left(u^{\varepsilon}\right)+c^{\varepsilon} \nabla \varphi^{\varepsilon}+\beta^{\varepsilon} \nabla^{2} \phi^{\varepsilon}, \\
& D^{2, \varepsilon}=\alpha^{\varepsilon^{T}} e\left(u^{\varepsilon}\right)+\beta^{\varepsilon^{T}} \nabla \varphi^{\varepsilon}+\gamma^{\varepsilon} \nabla^{2} \phi^{\varepsilon} .
\end{aligned}
$$

Depending on the type of electric loading, three different models indexed by $p$ appear at the limit. This result extends our previous study in [23] and shows that gradient theory broadens the understanding of sensors and actuators. When $p=2$ and $p=3$ we are able to express the constitutive laws as a Schur complement of the second order piezoelectric tensor in a framework valid for any symmetry class, which means that we do not make any simplifying assumptions dealing with the crystal symmetry of the material constituting the plate. When $p=1$, we are not able to explicitly derive the constitutive law of the limit model. Therefore, as in the case of first order piezoelectric rods treated treated in the next section, it seems very likely to us that non-local terms appear in this delicate situation. The study of the influence of the crystal symmetries on our models for $p=2,3$ shows that 
even for second order piezoelectricity, an electromechanical switch-off may appear in the structure if the plate is designed with specific materials.

\subsection{Piezoelectric slender rods}

From a technological point of view, piezoelectric materials can also be used in wires or slender rods. Now, the reference configuration of the piezoelectric structure is $\Omega^{\varepsilon}=$ $\varepsilon \times(0, L)$ with $L$ a fixed positive real number. The equations describing the equilibrium of the structure are the same as in the section 2.1 but of course the geometry of the various boundaries is different: we assume that $\Gamma_{m D}^{\varepsilon}=\varepsilon \omega \times\{0, L\}$.

To get our simplified models, we proceed as in the case of plates. Due to classical assumptions on the mechanical loading (which permits the justification of Bernoulli-Navier theory of elastic slender rods (see [11] and [16]) and on electrical loading:

$$
\left\{\begin{array}{l}
\text { if } p=1 \text { the extension of } \varphi_{0}^{\varepsilon} \text { into } \Omega^{\varepsilon} \text { does not depend on } \hat{x} \text { and } \Gamma_{e D}^{\varepsilon} \subset \varepsilon \omega \times\{0, L\} \\
\text { if } p=2 \text { there exists } \gamma_{e} \subset \partial \omega \text { such that } \Gamma_{e D}^{\varepsilon} \subset \varepsilon \times(0, L) .
\end{array}\right.
$$

the scaling is defined by:

$$
\left\{\begin{array}{l}
x=\left(\hat{x}, x_{3}\right) \in \bar{\Omega}=\omega \times(0, L) \mapsto \Pi^{\varepsilon} x=\left(\varepsilon \hat{x}, x_{3}\right) \in \bar{\Omega}^{\varepsilon} \\
s_{p}(\varepsilon)=S_{p}(\varepsilon) s^{\varepsilon} \\
\left(\hat{u}(\varepsilon)(x), u_{3}(\varepsilon)(x), \varphi(\varepsilon)(x)\right)=\left(\hat{u}^{\varepsilon}\left(\Pi^{\varepsilon} x\right), \varepsilon^{-1} u_{3}^{\varepsilon}\left(\Pi^{\varepsilon} x\right), \varepsilon^{-p} \varphi^{\varepsilon}\left(\Pi^{\varepsilon} x\right)\right)
\end{array}\right.
$$

so that $s_{p}(\varepsilon)$ is the unique solution of the variational problem

Find $s_{p}(\varepsilon) \in\left(0, \varphi_{0}\right)+V$ such that $\int_{\Omega} M(x) k_{p}(\varepsilon, s(\varepsilon)) \cdot k_{p}(\varepsilon, r)=L(r), \forall r \in V$, with now:

$$
k_{p}(\varepsilon,(v, \psi))=\left(\left(\varepsilon^{2} e_{\alpha \beta}(v), \varepsilon e_{\alpha 3}(v), e_{33}(v)\right),\left(\varepsilon^{p-2} \hat{\nabla} \psi, \varepsilon^{p-1} \partial_{3} \psi\right)\right) .
$$

As in the case of purely elastic slender rods (cf. [11]), finding the limit is a little bit more difficult and the limit problems are as follows:

$\overline{\mathcal{R}}(\Omega)_{1}\left\{\begin{array}{l}\text { Find }(\bar{u}, \bar{v}, \bar{w}, \bar{\phi}, \bar{\psi}) \in V_{1} \text { such that } \\ \int_{\Omega} M(x) k_{1}(\bar{u}, \bar{v}, \bar{w}, \bar{\phi}, \bar{\psi}) \cdot k_{1}\left(u^{\prime}, v^{\prime}, w^{\prime}, \phi^{\prime}, \psi^{\prime}\right) d x=L\left(u^{\prime}\right), \forall\left(u^{\prime}, v^{\prime}, w^{\prime}, \phi^{\prime}, \psi^{\prime}\right) \in V_{1},\end{array}\right.$ with

$$
\left\{\begin{array}{l}
V_{1}=V_{B N}(\Omega) \times R_{b}(\Omega) \times R D_{2}^{\perp} \times \Phi \times \Psi, \\
V_{B N}(\Omega)=\left\{v \in H_{\Gamma_{m}}^{1}(\Omega)^{3} ; e_{\alpha \beta}(v)=e_{\alpha 3}(v)=0\right\}, \\
R_{b}(\Omega)=\left\{v ; \exists c \in H_{0}^{1}(0, L) ; \widehat{v}(x)=c\left(x_{3}\right)\left(-x_{2}, x_{1}\right), v_{3} \in L^{2}\left(0, L ; H_{m}^{1}(\omega)\right)\right\}, \\
H_{m}^{1}(\omega)=\left\{v \in H^{1}(\omega) ; \int_{\omega} \psi(\widehat{x}) d \widehat{x}=0\right\}, \\
R D_{2}^{\perp}(\Omega)=\left\{\omega ; \widehat{w} \in L^{2}\left(0, L ; H_{m}^{1}(\omega)^{2}\right), w_{3}=0\right. \text { and } \\
\left.\quad \int_{\omega}\left(-x_{2} w_{1}\left(\widehat{x}, x_{3}\right)+x_{1} w_{2}\left(\widehat{x}, x_{3}\right)\right) d \widehat{x}=0, \text { ae } x_{3} \in(0, L)\right\}, \\
\Phi=\left\{\phi \in H_{0}^{1}(0, L) ; \phi(x)=\phi\left(x_{3}\right)\right\}, \\
\Psi=L^{2}\left(0, L ; H_{m}^{1}(\omega)\right), \\
k_{1}(u, v, w, \phi, \psi)=\left(\widehat{e}(w), e_{\alpha 3}(v), e_{33}(u), \widehat{\nabla} \psi, \frac{d \phi}{d x_{3}}\right)
\end{array}\right.
$$


and

$$
\overline{\mathcal{R}}(\Omega)_{2}\left\{\begin{array}{l}
\text { Find }(\bar{u}, \bar{v}, \bar{w}, \phi) \in V_{2} \text { such that } \\
\int_{\Omega} M(x) k_{2}(\bar{u}, \bar{v}, \bar{w}, \phi) \cdot k_{2}\left(u^{\prime}, v^{\prime}, w^{\prime}, \phi^{\prime}\right) d x=L\left(u^{\prime}\right), \forall\left(u^{\prime}, v^{\prime}, w^{\prime}, \phi^{\prime}\right) \in V_{2},
\end{array}\right.
$$

with

$$
\left\{\begin{array}{l}
V_{2}=V_{B N}(\Omega) \times R_{b}(\Omega) \times R D_{2}^{\perp} \times L^{2}\left(0, L ; H_{\gamma_{e}}^{1}(\omega)\right), \\
k_{2}(u, v, w, \phi)=\left(\widehat{e}(w), e_{\alpha 3}(v), e_{33}(u), \widehat{\nabla} \phi\right) .
\end{array}\right.
$$

The space $V_{B N}(\Omega)$ is the Bernoulli-Navier displacements space.

Of course, our proposal of model is obtained by taking the inverse scaling, that is to say a transported problem $\overline{\mathcal{R}}\left(\Omega^{\varepsilon}\right)_{p}$ posed over $\Omega^{\varepsilon}$. On the contrary to the case of plates, the state variables of the model do not reduce to the couple displacement/electrical potential but involve additional variables: two fields of displacements (easy to interpret mechanically) and a scalar field of electrical nature. Nevertheless, the kinematics of the state variables is simpler than the one of the genuine three-dimensional model which is very favourable from a numerical point of view. As in the purely elastic case it is worthwhile to note that for particular classes of monoclinic materials the additional variables $v, w$ and $\psi$ disappear [18]. Anyway, in the case $p=1$, the additional variables can be eliminated but it leads to non standard equations involving non local terms.

\subsection{Dynamical response of piezoelectric plates}

The interest of an efficient modeling of the dynamic response of piezoelectric plates lies in the fact that a major technological application of piezoelectric effects is the control of vibrations of structures through very thin plates or patches. We present two modelings depending on the various extents to which the magnetic effects are taken into account. Actually, because of the large discrepancy between the celerities of the mechanical and electromechanical waves, magnetic effects can be disregarded. That is why first we propose a modeling in the appropriate framework of the quasi-electrostatic approximation which claims that the electrical field still derives from an electrical potential.

\subsubsection{Quasi-electrostatic case}

Now a new parameter appears: the density $\rho$ of the plate. In the framework of the realistic quasi-electrostatic approximation, the electrical equilibrium equation remains true but the mechanical equilibrium equation is replaced by

$$
\operatorname{div} \sigma^{\varepsilon}+f^{\varepsilon}=\rho \ddot{u}^{\varepsilon} \text { in } \Omega^{\varepsilon}
$$

where the upper dot denotes the differentiation with respect to time. Under mild assumptions on the initial state and the essential assumption

$$
\int_{-1}^{+1} x_{3} \widetilde{M}_{1}\left(x_{1}, x_{2}, x_{3}\right) d x_{3}, \widetilde{M}_{2} \text { independent of } x_{3}
$$

it is possible to proceed to the study of the convergence of $s_{p}^{\varepsilon}$ when $\varepsilon$ goes to zero ([20], $[21])$, the result depends strongly on the relative behaviour of $\varepsilon$ and $\rho$. A unified accurate and simplified modeling is then obtained by simply adding $\int_{\Omega^{\varepsilon}} \rho \ddot{\bar{u}}_{p}^{\varepsilon} d x$ to the left hand side of the equation defining the descaled limit problem $\overline{\mathcal{P}}\left(\Omega^{\varepsilon}\right)_{p}$. Thus the relationship between 
the reduced stress, electric displacement, strain and gradient of electrical potential remains the same as in the static case: $\widetilde{M}_{p}^{\varepsilon}$ really describes the constitutive equations of the plate! The displacement fields involved in our simplified modeling being of Kirchoff-Love type, clearly four cases, indexed by $q$, of relative behaviours of the parameters determine the essential nature of the limit response of the plate to the electromechanical loading:

$$
\begin{aligned}
& q=1 \quad: \quad \rho \rightarrow \bar{\rho} \in(0,+\infty) \quad, \quad q=2 \quad: \quad \rho \rightarrow 0 \text { and } \rho / \varepsilon^{2} \rightarrow \infty \\
& q=3 \quad: \quad \rho / \varepsilon^{2} \rightarrow \overline{\bar{\rho}} \in(0,+\infty) \quad, \quad q=4 \quad: \quad \rho=o\left(\varepsilon^{2}\right) .
\end{aligned}
$$

In the cases $q=2$ and $q=4$, the limit response of the plate to the electromechanical loading is essentially quasi-static, while the cases $q=1$ and $q=3$ involve the acceleration of the displacement. Moreover, because of the assumption (17), appears a decoupling between the membrane motion and the flexural one. If $q=1,2$, the flexion is neglectible and the membrane response is dynamic if $q=1$, quasi-static if $q=2$. When $q=3,4$, the membrane response is quasi-static whereas the flexural response is dynamic if $q=3$ and quasi-static if $q=4$. In these last two cases, the equation giving the flexion does not involve the limit electric potential if $p=1$. The uncoupled elliptic and hyperbolic involved problems are two-dimensional and set on $\omega$.

The steps of the derivation of our model are the following. Firts we proceed to the same scaling as in section 2.1 and to a decomposition $s(\varepsilon)=s(\varepsilon)_{e}+s(\varepsilon)_{r}$, where $s(\varepsilon)_{e}$ solves a problem like $\mathcal{P}(\varepsilon, \Omega)_{p}$ and consequently whose asymptotic behavior is provided by section 2.1. Hence $s(\varepsilon)_{r}=\left(u(\varepsilon)_{r}, \varphi(\varepsilon)_{r}\right)$ satisfies an homogeneous variational evolution equation. Because the time derivatives do not act on $\varphi(\varepsilon)_{r}$, it is possible to exhibit a linear evolution equation for $u(\varepsilon)_{r}$ governed by a maximal monotone operator in a suitable Hilbert space whose norm depends on $(\varepsilon, \rho)$. Since the Trotter results of convergence of semi-goups of linear operators acting on variables spaces claim that the study of convergence of the transient problems reduces to the static case, the asymptotic behavior of $u(\varepsilon)_{r}$, and consequently of $s(\varepsilon)_{r}$ is easily determined by straightforward variants of the convergence results of the section 2.1 .

\subsubsection{The fully dynamic case}

In the previous case, the electrical field $E^{\varepsilon}$ was assumed to be curl-free and, consequently, equal to the gradient of the so-called electrical potential $\varphi^{\varepsilon}$. If we want to take into account the magnetic effects, the state of the plate is now described by a triplet $z^{\varepsilon}=\left(u^{\varepsilon}, E^{\varepsilon}, H^{\varepsilon}\right)$ where $H^{\varepsilon}$ is the magnetic field and the equations of the problem read as:

$$
\left\{\begin{array}{l}
\operatorname{div} \sigma^{\varepsilon}+f^{\varepsilon}=\rho \ddot{u}^{\varepsilon} \text { in } \Omega^{\varepsilon} \\
\dot{D}^{\varepsilon}=c \operatorname{curl} H^{\varepsilon} \text { in } \Omega^{\varepsilon} \\
\mu \dot{H}^{\varepsilon}=-c \operatorname{curl} E^{\varepsilon} \text { in } \Omega^{\varepsilon} \\
\left(\sigma^{\varepsilon}, D^{\varepsilon}\right)=M^{\varepsilon}\left(e\left(u^{\varepsilon}\right), E^{\varepsilon}\right) \text { in } \Omega^{\varepsilon}
\end{array}\right.
$$

with two kind of boundary conditions intimately linked to those of the previous cases (and, then, still indexed by $\mathrm{p}$ !):

$$
p=1: H^{\varepsilon} \wedge n^{\varepsilon}=j^{\varepsilon} \text { on } \partial \Omega^{\varepsilon}, p=2: E^{\varepsilon} \wedge n^{\varepsilon}=E_{0}^{\varepsilon} \wedge n^{\varepsilon} \text { on } \partial \Omega^{\varepsilon}
$$

Here $c, \mu, j^{\varepsilon}, E_{0}^{\varepsilon}$ stand for the light celerity, the magnetic permeability, the surface current density and the exterior electrical field respectively. We will assume that there exist sufficiently smooth fields $E_{0}, j$ such that: 


$$
\left\{\begin{array}{l}
E_{0}^{\varepsilon}\left(\Pi^{\varepsilon}\right)=\varepsilon^{2} E_{0}(x), \forall x \in \partial \Omega, \quad j^{\varepsilon}\left(\Pi^{\varepsilon}\right)=\varepsilon^{2} j(x), \forall x \in \Gamma_{ \pm} \\
j_{\alpha}^{\varepsilon}\left(\Pi^{\varepsilon} x\right)=\varepsilon j_{\alpha}(x), \quad j_{3}^{\varepsilon}\left(\Pi^{\varepsilon}\right)=\varepsilon^{2} j_{3}(x), \forall x \in \Gamma_{\text {lat }} .
\end{array}\right.
$$

Let

$$
\left\{\begin{array}{l}
\bar{E}_{1}^{\varepsilon}=\left\{E \in L^{2}\left(\Omega^{\varepsilon}\right)^{3} ; E_{3}=0, \partial_{3} E_{\alpha}=0\right\}, \\
\bar{E}_{2}^{\varepsilon}=\left\{E ; E_{3} \in L^{2}\left(\Omega^{\varepsilon}\right) ; \partial_{3}\left(\partial_{\alpha} E_{3}-\partial_{3} E_{\alpha}\right)=0, E_{\alpha}=0 \text { on } \Gamma_{ \pm}^{\varepsilon}\right\}, \\
\bar{H}_{1}^{\varepsilon}=\left\{H \in L^{2}\left(\Omega^{\varepsilon}\right)^{3} ; H_{\alpha}=0, \partial_{3} H_{3}=0\right\}, \\
\bar{H}_{2}^{\varepsilon}=\left\{H \in L^{2}\left(\Omega^{\varepsilon}\right)^{3} ; H_{3}=0, \partial_{3} H_{\alpha}=0\right\}, \\
\bar{Z}_{p}^{\varepsilon}=\bar{V}^{\varepsilon} \times \bar{E}_{p}^{\varepsilon} \times \bar{H}_{p}^{\varepsilon}, k_{1}(v, E)=\left(e_{\alpha \beta}(v), E_{\alpha}\right), k_{2}(v, E)=\left(e_{\alpha \beta}(v), E_{3}\right), 1 \leqslant \alpha, \beta \leqslant 3 .
\end{array}\right.
$$

Under (17), (19) and mild assumptions on the smoothness of the initial state, it can be shown that the state $z_{p}^{\varepsilon}$ is asymptotically equivalent to $\bar{z}_{p}^{\varepsilon}=\left(\bar{u}_{p}^{\varepsilon}, \bar{E}_{p}^{\varepsilon}, \bar{H}_{p}^{\varepsilon}\right)$ which satisfies:

$$
\left\{\begin{array}{l}
\int_{\Omega^{\varepsilon}} \rho \ddot{\bar{u}}_{p}^{\varepsilon} \cdot v d x+\int_{\Omega^{\varepsilon}} \widetilde{M}_{p}^{\varepsilon} k_{p}\left(\bar{u}_{p}^{\varepsilon}, \bar{E}_{p}^{\varepsilon}\right) \cdot k_{p}(v, 0) d x=L_{p}^{\varepsilon}(v, 0), \forall v \in V_{K L}\left(\Omega^{\varepsilon}\right) \\
\left(\dot{\bar{D}}_{1}^{\varepsilon}\right)_{1}(\widehat{x})=-c \partial_{2}\left(\bar{H}_{1}^{\varepsilon}\right)_{3}(\widehat{x})+j_{1}^{\varepsilon}(\widehat{x}, \varepsilon)+j_{1}^{\varepsilon}(\widehat{x},-\varepsilon), \forall \widehat{x} \in \omega \\
\left(\dot{\bar{D}}_{1}^{\varepsilon}\right)_{2}(\widehat{x})=c \partial_{1}\left(\bar{H}_{1}^{\varepsilon}\right)_{3}(\widehat{x})+j_{2}^{\varepsilon}(\widehat{x}, \varepsilon)+j_{2}^{\varepsilon}(\widehat{x},-\varepsilon), \forall \widehat{x} \in \omega \\
\left.\dot{\bar{D}}_{2}^{\varepsilon}\right)_{2}(\widehat{x})=c\left(\partial_{1}\left(\bar{H}_{2}^{\varepsilon}\right)_{2}-\partial_{2}\left(\bar{H}_{2}^{\varepsilon}\right)_{1}\right)(\widehat{x}), \forall \widehat{x} \in \omega \\
\mu\left(\dot{\bar{H}}_{1}^{\varepsilon}\right)_{3}(\widehat{x})=-c\left(\partial_{1}\left(\bar{E}_{1}^{\varepsilon}\right)_{2}-\partial_{2}\left(\bar{E}_{1}^{\varepsilon}\right)_{1}\right)(\widehat{x}), \forall \widehat{x} \in \omega \\
\mu\left(\dot{\bar{H}}_{2}^{\varepsilon}\right)_{1}(\widehat{x})=-c\left(\partial_{2}\left(\bar{E}_{2}^{\varepsilon}\right)_{3}-\partial_{3}\left(\bar{E}_{2}^{\varepsilon}\right)_{2}\right)(\widehat{x}), \forall \widehat{x} \in \omega \\
\mu\left(\dot{\bar{H}}_{2}^{\varepsilon}\right)_{2}(\widehat{x})=-c\left(\partial_{3}\left(\bar{E}_{2}^{\varepsilon}\right)_{1}-\partial_{1}\left(\bar{E}_{2}^{\varepsilon}\right)_{3}\right)(\widehat{x}), \forall \widehat{x} \in \omega \\
\left(\bar{\sigma}_{p}^{\varepsilon}, \bar{D}_{p}^{\varepsilon}\right)=\widetilde{M}_{p}^{\varepsilon} k_{p}\left(\bar{u}_{p}^{\varepsilon}, \bar{E}_{p}^{\varepsilon}\right)
\end{array}\right.
$$

with the boundary conditions:

$$
\bar{H}_{1}^{\varepsilon} \wedge n^{\varepsilon}=\frac{1}{2 \varepsilon} \int_{-\varepsilon}^{+\varepsilon} j^{\varepsilon}\left(\cdot, x_{3}\right) d x_{3} \text { on } \Gamma_{\text {lat }}^{\varepsilon}, \bar{E}_{2}^{\varepsilon} \wedge n^{\varepsilon}=\bar{E}_{0}^{\varepsilon} \wedge n^{\varepsilon} \text { on } \Gamma_{ \pm}^{\varepsilon} .
$$

The structure of the equations of our model is the same that those of the genuine model, but the problems are two-dimensional and with a lesser number of degrees of freedom for the state fields!

Again, the key-point is to formulate a suitable scaling of the problems in terms of an evolution equation governed by a maximal monotone operator in an Hilbert space of possible states with finite scaled energy. By using Trotter theory we only have to consider the limit behavior of a perturbation of the variational equation which defines $\mathcal{P}(\varepsilon, \Omega)_{p}$. This perturbation taking into account a scaling of the curl operator, the limit behavior is obtained by using weak continuity and integration by parts in the terms involving the curl operator.

\section{Mathematical modelings of smart structures}

The essential technological interest of piezoelectric devices being the monitoring of a deformable body they are bonded to or integrated in, this section is devoted to smart structures. Here we intend to propose various asymptotic models for the behavior of the body through the study of the system constituted by a very thin linearly piezoelectric flat patch 
perfectly bonded to or integrated in a linearly elastic or piezoelectric three dimensional body.

\subsection{Piezoelectric patches}

A reference configuration for the body is an open set $\Omega$ laying in $\left\{x_{3}<0\right\}$ whose part of its Lipschitz-continuous boundary $\partial \Omega$ is a non-empty domain $S$ in $\left\{x_{3}=0\right\}$ and such that $S \times(-L, 0)$ is included in $\Omega$ for some positive real number $L$, while the patch occupies $B^{\varepsilon}:=S \times(0, \varepsilon), \varepsilon$ being a small real number; let $\mathcal{O}^{\varepsilon}:=\Omega \cup S \cup B^{\varepsilon}$. The body is clamped on a part $\Gamma_{0}$ of $\partial \Omega \backslash S$ with a positive two-dimensional measure $\mathcal{H}_{2}\left(\Gamma_{0}\right)$, and subjected to body forces and surface forces on $\Gamma_{1}:=\partial \Omega \backslash\left(S \cup \Gamma_{0}\right)$ of densities $f$ and $F$. Moreover, for all $\delta$ in $\mathbb{R}$, let $S^{\delta}$ denotes $S+\delta e_{3},\left\{e_{1}, e_{2}, e_{3}\right\}$ being a basis of the Euclidean physical space assimilated to $\mathbb{R}^{3}$, surface forces of density $G$ acts on $S^{\varepsilon}$ whilst the patch is free of mechanical loading and electric charges in $B^{\varepsilon}$ and on its lateral boundary $\partial S \times(0, \varepsilon)$. If $u^{\varepsilon}, e\left(u^{\varepsilon}\right), \sigma^{\varepsilon}$ denote the fields of displacement, strain and stress in $\mathcal{O}^{\varepsilon}$ and $\varphi^{\varepsilon}, D^{\varepsilon}$ stand for the electric potential and the electric displacement, part of the equations describing the electromechanical equilibrium read as:

$$
\left\{\begin{array}{l}
\operatorname{div} \sigma^{\varepsilon}=\tilde{f} \text { in } \mathcal{O}^{\varepsilon}, u^{\varepsilon}=0 \text { on } \Gamma_{0}, \\
\sigma^{\varepsilon} n=F \text { on } \Gamma_{1}, \sigma^{\varepsilon} n=G^{\varepsilon} \text { on } S^{\varepsilon}, \sigma^{\varepsilon} n=0 \text { on } \partial S \times(0, \varepsilon), \\
\operatorname{div} D^{\varepsilon}=0 \text { in } B^{\varepsilon}, D^{\varepsilon} \cdot n=0 \text { on } \partial S \times(0, \varepsilon), \\
\sigma^{\varepsilon}=a e\left(u^{\varepsilon}\right) \text { in } \Omega,\left(\sigma^{\varepsilon}, D^{\varepsilon}\right)=\frac{1}{\varepsilon} M\left(e\left(u^{\varepsilon}\right), \nabla \varphi^{\varepsilon}\right) \text { in } B^{\varepsilon},
\end{array}\right.
$$

$\tilde{f}$ is the extension of $f$ to $B^{\varepsilon}$ by $0, n$ is the unit outward normal and $a$ denotes the elasticity tensor which satisfies

$$
a \in L^{\infty}\left(\Omega ; \mathcal{L}\left(\mathbb{S}^{3}\right)\right), \quad \exists c ; c|e|^{2} \leq a(x) e \cdot e, \quad \forall e \in \mathbb{S}^{3}, \text { a.e. } x \in \Omega,
$$

while $M$ is an element of $L^{\infty}(S ; \mathcal{L}(\mathbb{H}))$ satisfying

$$
M=\left[\begin{array}{cc}
\alpha & -\beta \\
\beta^{T} & \gamma
\end{array}\right] \quad, \quad \exists \kappa>0 ; \quad \kappa|h|^{2} \leq M h \cdot h, \quad \forall h \in \mathbb{H} \text {, a.e. } x \in S .
$$

The models will be distinguished according to the additional necessary boundary conditions on $S^{\varepsilon}$ and $S$, characterized by an index $p$ in $\{1,2\}^{2}$. Case $p_{1}=1$ corresponds to a condition for the electric displacement on $S^{\varepsilon}$ :

$$
D^{\varepsilon} \cdot n=q^{\varepsilon} \quad \text { on } S^{\varepsilon}
$$

$q^{\varepsilon}$ being a density of electrical charges, while $p_{1}=2$ corresponds to a condition of given electrical potential:

$$
\varphi^{\varepsilon}=\varphi_{0}^{\varepsilon} \quad \text { on } S^{\varepsilon}
$$

roughly speaking, $p_{1}=1$ deals with patches used as sensors whereas $p_{1}=2$ concerns actuators (see [19], [20]). Index $p_{2}$ accounts for the status of the interface between the patch and the body: $p_{2}=1$ corresponds to an insulating interface, $p_{2}=2$ corresponds to a grounded interface:

$$
\begin{aligned}
D^{\varepsilon} \cdot n=0 & \text { on } S, \\
\varphi^{\varepsilon}=0 & \text { on } S .
\end{aligned}
$$


Introducing the transverse average of the strain and of the electrical field, it is easy to go to the limit as $\varepsilon$ goes to 0 and to show that the limit model corresponds to purely mechanical reinforcement problem along $S$.

Moreover, when $p=(1,2)$ or $p=(2,1)$, the electric data $q$ or $\varphi_{0}$ does not have any influence on the limit model which corresponds to a purely elastic surface reinforcement of the body. However, the characteristics of this reinforcement may depend on the dielectric or piezoelectric coefficients (see [6]). On the contrary, electrical data $q$ or $\varphi_{0}$ plays a role in models $(1,1)$ or $(2,2)$. More precisely, $f, F$ and $G$ being fixed, there is a one-to-one mapping between the applied electrical potential and the limit displacement. It is thus "theoretically" possible to determine what could be the electrical potential to apply on $S^{\varepsilon}$ in order to get a desired displacement. An approximate procedure may be done easily by finite elements. Another application is that the patch may shift the spectrum of the body in an interesting way, that is why we may regard the patch as an actuator. When $p=(1,1)$ there is also a one-to-one mapping between the limit displacement and the electrical charges. Thus the measurement of the latter may supply the knowledge of the state of displacements: the patch acts as a sensor!

\subsection{Piezoelectric junctions}

In this section, we first present our results dealing with smart structures composed of materials whose coefficients are of the same order of magnitude. However, as it is often observed that the electric permeability is very small compared to other coefficient, next we carry out a general study of piezoelectric junctions whose material coefficients are of different order of magnitude.

\subsection{Piezoelectric junctions with material coefficients of same mag- nitude}

In this section, we present various asymptotic models, indexed by $p=\left(p_{1}, p_{2}\right) \in\{1,2,3,4\}^{2}$, for a thin piezoelectric junction between two linearly piezoelectric $\left(p_{2}=1\right)$ or elastic $\left(p_{2}>1\right)$ bodies. Index $p_{1}$ is relative to the magnitude of the piezoelectric coefficients of the adhesive, characterized by a single parameter $\mu$, with respect to that of the constant thickness $2 \varepsilon$ of a layer containing the adhesive. More precisely, we assume that $h:=(\varepsilon, \mu)$ takes values in a countable set with a sole cluster point $\bar{h} \in\{0\} \times[0,+\infty]$ so that:

$$
\left\{\begin{array}{l}
p_{1}=1: \bar{\mu}_{1}:=\lim _{h \rightarrow \bar{h}}(\varepsilon \mu) \in(0,+\infty) \\
p_{1}=2: \bar{\mu}_{1}:=\lim _{h \rightarrow \bar{h}}(\varepsilon \mu)=0, \quad \bar{\mu}_{2}:=\lim _{h \rightarrow \bar{h}}(\mu / 2 \varepsilon)=+\infty \\
p_{1}=3: \bar{\mu}_{2}:=\lim _{h \rightarrow \bar{h}}(\mu / 2 \varepsilon) \in(0,+\infty) \\
p_{1}=4: \quad \bar{\mu}_{2}:=\lim _{h \rightarrow \bar{h}}(\mu / 2 \varepsilon)=0 .
\end{array}\right.
$$

As previously said, index $p_{2}$ characterizes the status of the adherents but also that of the interfaces between adherents and adhesive:

$$
\left\{\begin{array}{l}
p_{2}=1: \text { the two interfaces are electromechanically perfectly permeable } \\
p_{2}=2: \text { the two interfaces are electrically impermeable, } \\
p_{2}=3: \text { one interface is electrically impermeable while the other is electroded, } \\
p_{2}=4: \text { the two interfaces are electroded. }
\end{array}\right.
$$

Let $\Omega$ be a domain, with Lipschitz-continuous boundary, whose intersection $S$ with $\left\{x_{3}=0\right\}$ is a domain of $\mathbb{R}^{2}$ of positive two-dimensional Hausdorff measure $\mathcal{H}_{2}(S)$. Let 
$\Omega_{ \pm}:=\Omega \cap\left\{ \pm x_{3}>0\right\}$ and $\varepsilon$ be a small positive number, then adhesive and adherents occupy $B^{\varepsilon}:=S \times(-\varepsilon, \varepsilon), \Omega_{ \pm}^{\varepsilon}:=\Omega_{ \pm} \pm \varepsilon e_{3}$, respectively; let $\Omega^{\varepsilon}=\Omega_{+}^{\varepsilon} \cup \Omega_{-}^{\varepsilon}, S_{ \pm}^{\varepsilon}:=S \pm \varepsilon e_{3}$, $\mathcal{O}^{\varepsilon}:=\Omega^{\varepsilon} \cup B^{\varepsilon} \cup_{ \pm} S_{ \pm}^{\varepsilon}$. Let $\left(\Gamma_{\mathrm{mD}}, \Gamma_{\mathrm{mN}}\right),\left(\Gamma_{\mathrm{eD}}, \Gamma_{\mathrm{eN}}\right)$ be two partitions of $\partial \Omega$ with $\mathcal{H}_{2}\left(\Gamma_{\mathrm{mD}}\right)$, $\mathcal{H}_{2}\left(\Gamma_{\mathrm{eD}}\right)>0$ and $0<\delta:=\operatorname{dist}\left(\Gamma_{\mathrm{eD}}, S\right)$. For all $\Gamma$ in $\left\{\Gamma_{\mathrm{mD}}, \Gamma_{\mathrm{mN}}, \Gamma_{\mathrm{eD}}, \Gamma_{\mathrm{eN}}\right\}, \Gamma_{ \pm}, \Gamma_{ \pm}^{\varepsilon}, \Gamma^{\varepsilon}$ denotes $\Gamma \cap\left\{ \pm x_{3}>0\right\}, \Gamma_{ \pm} \pm \varepsilon e_{3}, \cup_{ \pm} \Gamma_{ \pm}^{\varepsilon}$, respectively; if $\left(\gamma_{\mathrm{D}}, \gamma_{\mathrm{N}}\right)$ is a partition of $\gamma:=\partial S$, we denote $\left\{\gamma_{\mathrm{D}}, \gamma_{\mathrm{N}}, \gamma\right\} \times(-\varepsilon, \varepsilon)$ by $\left\{\Gamma_{\mathrm{DI}}^{\varepsilon}, \Gamma_{\mathrm{NI}}^{\varepsilon}, \Gamma_{\text {lat }}^{\varepsilon}\right\}$. The structure made of the adhesive and the two adherents, perfectly stuck together along $S_{ \pm}^{\varepsilon}$, is clamped on $\Gamma_{\mathrm{mD}}^{\varepsilon}$, subjected to body forces of density $f^{\varepsilon}$ and to surface forces of density $F^{\varepsilon}$ on $\Gamma_{m N}^{\varepsilon}$ and vanishing on $\Gamma_{\text {lat }}^{\varepsilon}$. Moreover, a given electric potential $\varphi_{p_{0}}^{h}$ is applied on $\Gamma_{\mathrm{DI}}^{\varepsilon}$ and, when $p_{2}=1$, on $\Gamma_{\mathrm{eD}}^{\varepsilon}$, while electric charges of density $d^{\varepsilon}$ appear on $\Gamma_{\mathrm{NI}}^{\varepsilon}$ and, when $p_{2}=1$, on $\Gamma_{\mathrm{eN}}^{\varepsilon}$.

If $\sigma_{p}^{h}, u_{p}^{h}, e\left(u_{p}^{h}\right), D_{p}^{h}, \varphi_{p}^{h}$ stand for the fields of stress, displacement, strain, electric displacement and electric potential, respectively, the constitutive equations of the structure, for all $p_{1}$ in $\{1,2,3,4\}$, read as:

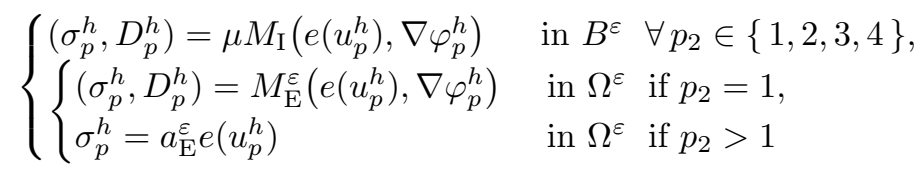

where

$$
\left(M_{\mathrm{E}}^{\varepsilon}, a_{\mathrm{E}}^{\varepsilon}\right)(x)=\left(M_{\mathrm{E}}, a_{\mathrm{E}}\right)\left(x \mp \varepsilon e_{3}\right) \quad \forall x \in \Omega_{ \pm}^{\varepsilon}
$$

$$
\left\{\begin{array}{l}
\left(M_{\mathrm{I}}, M_{\mathrm{E}}\right) \in L^{\infty}(S \times \Omega ; \mathcal{L}(\mathbb{H})) \text { such that } \\
M_{\mathrm{P}}=\left[\begin{array}{cc}
a_{\mathrm{P}} & -b_{\mathrm{P}} \\
b_{\mathrm{P}}^{T} & c_{\mathrm{P}}
\end{array}\right] ; \\
\exists \kappa>0, \quad \kappa|k|^{2} \leq M_{\mathrm{P}}(x) k \cdot k, \quad \forall k \in \mathbb{H}:=\mathbb{S}^{3} \times \mathbb{R}^{3}, \text { a.e. } x \in \Omega, \forall \mathrm{P} \in\{\mathrm{I}, \mathrm{E}\} .
\end{array}\right.
$$

Lastly we have to add the following conditions on $S_{ \pm}^{\varepsilon}$ :

$$
\left\{\begin{array}{lll}
p_{2}=2 & D_{p}^{h} \cdot e_{3}=0 & \text { on } S_{ \pm}^{\varepsilon} \\
p_{2}=3 & D_{p}^{h} \cdot e_{3}=0 & \text { on } S_{+}^{\varepsilon}, \quad \varphi_{p}^{h}=\varphi_{p_{0}}^{h} \text { on } S_{-}^{\varepsilon} \\
p_{2}=4 & \varphi_{p}^{h}=\varphi_{p_{0}}^{h} & \text { on } S_{ \pm}^{\varepsilon}
\end{array}\right.
$$

the electric potential $\varphi_{p_{0}}^{h}$ being given on $S_{+}^{\varepsilon}$ or $S_{ \pm}^{\varepsilon}$.

The same averaging method through the junction easily leads to our limit models. We are then able to show that in the case of piezoelectric adhesive and adherents $\left(p_{2}=1\right)$, our results extend those obtained in elasticity (see $[1,5]$ ). The asymptotic behavior of the adhesive strongly depends on the magnitude of the stiffness compared to that of the thickness. When the magnitude of the stiffness is of the order of the inverse of the thickness, the adhesive is replaced by a material piezoelectric surface perfectly bonded to the adherents. When it is lesser, the adhesive is replaced by an electromechanical constraint between the two adherents which can be perfect adhesion, electromechanical pull-back or free separation, according to the order of magnitude of the stiffness which is, respectively, larger, equal or lower than that of the thickness.

Similarly, in the case of a thin piezoelectric layer embedded between two elastic adherents, depending on the magnitude of the stiffness, the adhesive is replaced by a material elastic surface perfectly bonded to the adherents or by a mechanical constraint between the adherents. In the case of electrically impermeable interfaces, the material surface has a non local elastic behavior (see [7]), the constitutive equations being derived from the asymptotic behavior of a thin piezoelectric plate acting as a sensor (case $p=1$ in [23]). 
When one interface is electrically impermeable while the other is electroded, the material surface is an elastic membrane. When the two interfaces are electroded, the material surface is an elastic membrane with residual stress. In these last two cases, the constitutive equations are derived from the asymptotic behavior of a thin piezoelectric plate acting as an actuator (case $p=2$ in [23]). The mechanical constraint is perfect adhesion, elastic pull-back or free separation according to the order of magnitude of the stiffness. In the case of electrically impermeable interfaces, the elastic pull-back is of non local nature (since the state variable of electric nature $\phi$, additional to the relative displacement, can be eliminated). In the two other cases, the elastic pull-back is local. When the two interfaces are electroded, it is similar to the purely elastic case, while, if only one interface is electroded, piezoelectric and dielectric coefficients enter the limit constitutive equations.

\subsubsection{Piezoelectric hybrid junctions}

Due to the wide range of values taken by the elastic, piezoelectric and dielectric coefficients of various devices, it is worthwhile to extend our previous study [7] devoted to thin linearly piezoelectric junctions to the case when the elastic, piezoelectric and dielectric coefficients of the junction are not of the same order of magnitude. Our various asymptotic models for a thin piezoelectric junction between two linearly piezoelectric or elastic bodies will be indexed by $p=\left(p_{1}, p_{2}, p_{3}\right)$ in $\{1,2,3,4\}^{3}$. Indices $p_{1}$ and $p_{2}$ are respectively relative to the magnitude of the elastic and dielectric coefficients of the adhesive with respect to that of the constant thickness $2 \varepsilon$ of the layer containing the adhesive. More precisely, we assume that $h:=(\varepsilon, \mu)=\left(\varepsilon, \mu_{m m}, \mu_{e e}, \mu_{m e}\right)$ takes values in a countable set with a sole cluster point $\bar{h} \in\{0\} \times[0,+\infty]^{3}$, so that

$$
\begin{gathered}
\left\{\begin{aligned}
p_{1}=1: \bar{\mu}_{m m}^{1}:=\lim _{h \rightarrow \bar{h}}\left(2 \varepsilon \mu_{m m}\right) \in(0,+\infty) \\
p_{1}=2: \bar{\mu}_{m m}^{1}:=\lim _{h \rightarrow \bar{h}}\left(2 \varepsilon \mu_{m m}\right)=0, \\
\bar{\mu}_{m m}^{2}:=\lim _{h \rightarrow \bar{h}}\left(\mu_{m m} / 2 \varepsilon\right)=+\infty \\
p_{1}=3: \bar{\mu}_{m m}^{2}:=\lim _{h \rightarrow \bar{h}}\left(\mu_{m m} / 2 \varepsilon\right) \in(0,+\infty) \\
p_{1}=4: \bar{\mu}_{m m}^{2}:=\lim _{h \rightarrow \bar{h}}\left(\mu_{m m} / 2 \varepsilon\right)=0
\end{aligned}\right. \\
\left\{\begin{aligned}
p_{2}=1: \bar{\mu}_{e e}^{1}:=\lim _{h \rightarrow \bar{h}}\left(2 \varepsilon \mu_{e e}\right) \in(0,+\infty) \\
p_{2}=2: \bar{\mu}_{e e}^{1}:=\lim _{h \rightarrow \bar{h}}\left(2 \varepsilon \mu_{e e}\right)=0, \\
\bar{\mu}_{e e}^{2}:=\lim _{h \rightarrow \bar{h}}\left(\mu_{e e} / 2 \varepsilon\right)=+\infty \\
p_{2}=3: \bar{\mu}_{e e}^{2}:=\lim _{h \rightarrow \bar{h}}\left(\mu_{e e} / 2 \varepsilon\right) \in(0,+\infty) \\
p_{2}=4: \bar{\mu}_{e e}^{2}:=\lim _{h \rightarrow \bar{h}}\left(\mu_{e e} / 2 \varepsilon\right)=0 .
\end{aligned}\right.
\end{gathered}
$$

The parameters $\mu_{m m}, \mu_{e e}, \mu_{m e}$ respectively characterize the order of magnitude of the elastic, dielectric and piezoelectric coefficients of the adhesive. The case $p_{1}=p_{2}$, being already treated in [7], in the following we assume $p_{1} \neq p_{2}$. As in [7], index $p_{3}$ characterizes the status of the adherents but also that of the interfaces between adherents and adhesive:

$$
\left\{\begin{array}{l}
p_{3}=1: \text { the two interfaces are electromechanically perfectly permeable, } \\
p_{3}=2: \text { the two interfaces are electrically permeable, } \\
p_{3}=3: \text { one interface is electrically permeable while the other one bears an electrode, } \\
p_{3}=4: \text { the two interfaces bear an electrode. }
\end{array}\right.
$$


Therefore, the constitutive equations of the structure, for all $\hat{p}:=\left(p_{1}, p_{2}\right)$, read as:

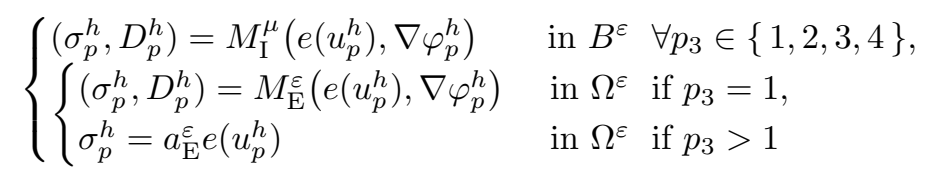

where

$$
\begin{aligned}
& \left(M_{\mathrm{E}}^{\varepsilon}, a_{\mathrm{E}}^{\varepsilon}\right)(x)=\left(M_{\mathrm{E}}, a_{\mathrm{E}}\right)\left(x \mp \varepsilon e_{3}\right) \quad \forall x \in \Omega_{ \pm}^{\varepsilon}
\end{aligned}
$$

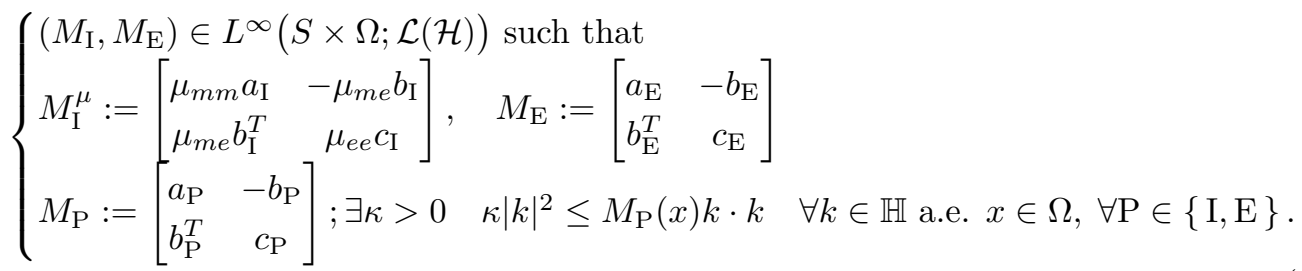

Lastly we have to add the following conditions on $S_{ \pm}^{\varepsilon}$ :

$$
\left\{\begin{array}{lll}
p_{3}=2 & D_{p}^{h} \cdot e_{3}=0 & \text { on } S_{ \pm}^{\varepsilon}, \\
p_{3}=3 & D_{p}^{h} \cdot e_{3}=0 & \text { on } S_{+}^{\varepsilon}, \quad \varphi_{p}^{h}=\varphi_{p_{0}}^{h} \text { on } S_{-}^{\varepsilon}, \\
p_{3}=4 & \varphi_{p}^{h}=\varphi_{p_{0}}^{h} & \text { on } S_{ \pm}^{\varepsilon},
\end{array}\right.
$$

the electric potential $\varphi_{p_{0}}^{h}$ being given on $S_{+}^{\varepsilon}$ or $S_{ \pm}^{\varepsilon}$.

Our results show that for piezoelectric adhesive and adherents, when the elastic and dielectric coefficients of the adhesive are not of the same order, the piezoelectric coupling remains in the asymptotic model only when $\hat{p}=(1,3)$ or $(3,1)$. More generally, when (necessarily only) one index $p_{1}$ or $p_{2}$ is equal to 1 , the status of the limit model for the adhesive is hybrid. When $p_{1}=1$, the adhesive is replaced by both a material surface perfectly bonded to the adherents, from the mechanical point of view, and a constraint, from the electrical point view. On the contrary when $p_{2}=1$, a mechanical constraint appears with an electrical material surface perfectly permeable. The mechanical material surface is an elastic membrane with a possible nonvanishing (only when $\hat{p}=(1,3)$ ) residual stress stemming from the possible discontinuity of the electrical potential induced by the limit electrical constraint which is perfect permeability, electric pull-back or impermeability, according to the magnitude of the dielectric coefficients. The electrical material surface is of linear conductor type with a possible nonvanishing (only when $\hat{p}=(3,1)$ ) residual term stemming from the possible nonvanishing relative displacement induced by the mechanical constraint which is perfect adhesion, elastic pull-back or free separation according to the magnitude of the stiffness of the adhesive. When both $p_{1}$ and $p_{2}$ are greater than 1 , the adhesive is replaced by an electromechanical constraint. As the orders of magnitude of the elastic and dielectric coefficients differ, this electromechanical constraint reduces to two independent mechanical and electrical constraints of the types previously evocated according to the values of $p_{1}$ and $p_{2}$, respectively.

For a thin piezoelectric layer embedded between two purely elastic adherents through two electrically impermeable interfaces, the piezoelectric coupling remains in the asymptotic model only when $\hat{p}=(1,3)$ or $(3,1)$. When $\hat{p}=(1,3)$ the adhesive layer is replaced by a piezoelectric material surface; when $\hat{p}=(3,1)$, it is replaced by a material conductive surface and a mechanical constraint. This constraint is of elastic pull-back type with a residual term stemming from the electrical potential in the conductive surface. Actually, when $p_{1}=1$, the adhesive layer is replaced by a material elastic surface perfectly bonded 
to the adherents. When $p_{2}=3$, the material surface has a non-local elastic behavior since the electrical potential can be eliminated, in the other cases the material surface is a standard elastic membrane. When $p_{1}$ ranges from 2 to 4 , the adhesive layer is replaced by a mechanical constraint which is perfect adhesion, elastic pull-back or free separation. The elastic pull-back is nonlocal when $p_{2}=1$. When $p_{2}=2$, the electric potential vanishes, in the remaining cases the limit surface is a linear elastic conductor.

The limit models for a thin piezoelectric layer embedded between two elastic adherents, through either two electroded interfaces or one electroded and the other being impermeable, only differ when $\hat{p}=(1,3)$. In all cases, there is a perfect decoupling between Electricity and Mechanics. When the magnitude of the stiffness is of the order of the inverse of the thickness, the adhesive is replaced by an elastic material membrane perfectly bonded to the adherents; when it is lesser, the adhesive is replaced by a mechanical constraint which is perfect adhesion, elastic pull-back, free separation according to the magnitude of the stiffness. The limit surface is at a given applied potential when $\hat{p} \in\{3,4\} \times\{1\}$, at a vanishing one in the other cases. Actually when $p=(1,3,3)$, the memory of electricity remains because piezoelectric and dielectric coefficients enter in the constitutive equations of the elastic membrane the adhesive layer reduces to.

Eventually the previous method may work when the elastic and dielectric coefficients of the junction are of the same order of magnitude with piezoelectric coefficients of lesser order. Obviously the conclusions of [7] remain but with $b_{I}$ replaced by 0 , so that the piezoelectric coupling disappears in the asymptotic models.

\section{References}

[1] A. Aitmoussa, Modélisation et études des singularités de contraintes d'un joint collé très mince, Ph.D. thesis, Université Montpellier 2, France (1989)

[2] P. Bisegna, F. Maceri, A consistent theory of thin piezoelectric plates, J. Intelligent Material Systems and Structures, Vol. 7 (1996), 372-389.

[3] P.G. Ciarlet, Mathematical Elasticity, Vol. II, North-Holland, 1997.

[4] G. Geymonat, C. Licht, T. Weller, Plates made of piezoelectric materials: When are they really piezoelectric?, Applied Mathematical Modeling (2011), 35, 165173.

[5] C. Licht, Some new mathematical modelings of junctions, East-West Journal of Mathematics (2011), 13(1), 23-33.

[6] C. Licht, S. Orankitjaroen, P. Viriyasrisuwattana, T. Weller, Bonding a linearly piezoelectric patch on a linearly elastic body, C.R. Mécanique (2014), 342(4), 234-239

[7] C. Licht, S. Orankitjaroen, P. Viriyasrisuwattana, T. Weller, Thin linearly piezoelectric junctions, C.R. Mécanique (2015), 343(4), 282-288

[8] G.A. Maugin, D. Attou, An asymptotic theory of thin piezoelectric plates, Quat. J. Mech. Appl. Math., Vol. 43 (1990), 347 - 362.

[9] C. A. Mead, 1962. Electron transport mechanism in thin insulating films. Phys. Rev. 128, 20882095. 
[10] R. D. Mindlin, 1968. Polarization gradient in elastic dielectrics. Int. J. Solids Struct. 4, 637-642, 1968.

[11] F. Murat, A. Sili, Comportement asymptotique des solutions du système de l'élasticité linéarisée anisotrope hétérogène dans des cylindres minces, C. R. Acad. Sci. Paris, t. 328, Série I (199),179-184, 1999

[12] M. Rahmoune, A. Benjeddou, R. Ohayon, New thin piezoelectric plates models, J. Intelligent Material Systems and Structures, Vol. 9 (1998), 1017-1029.

[13] D. Royer, E. Dieulesaint, Ondes élastiques dans les solides, Tome I, Masson, 1996.

[14] A. Sène, Modélisation asymptotique de plaques : contrôlabilité exacte frontière, piezoélectricité, Thèse de l'Université Joseph Fourrier - Grenoble I, 2000.

[15] R. A. Toupin, 1956. The elastic dielectric. J. Rational Mech. Anal. 5, 849-915.

[16] L. Trabucho, J.M. Viano, Mathematical modelling of rods, Handbook of Numerical Analysis, Vol. IV, North-Holland, Amsterdam, 1996.

[17] W. Voigt, 1910. Lehrbuch der Kristallphysik. B. G. Teubner, Leipzig.

[18] D. Vrisysrisuwattana, C. Licht, T. Weller, S. Koonprasert, Mathematical modelling of piezoelectric thin plates and slender beams through functional analysis, The twelfth Annual Meeting in Mathematics 2007, Burapha University, Chonburi, Thaland, 17-18 may 2007, to appear.

[19] T. Weller, C. Licht, Analyse asymptotique de plaques minces linéairement piézoélectriques, C. R. Acad. Sci. Paris, Sér. I 335 (2002), 309-314.

[20] T. Weller, Étude des symétries et modèles de plaques en piézoélectrictité linéarisée, Thèse de l'Université Montpellier II, 2004.

[21] T. Weller, C. Licht, Réponse dynamique asymptotique de plaques minces linéairement piézoélectriques dans l'approximation quasi-électrostatique, C.R. Mecanique, 332 (2004), 519-524.

[22] T. Weller, C. Licht, Modeling of linearly electromagneto-elastic thin plates, C.R. Mecanique 335 (2007), 201-206.

[23] T. Weller, C. Licht, 2010. Asymptotic modeling of thin piezoelectric plates. Ann. Solid Struct. Mech. 1, 173-188. 